\title{
Path synthesis of a finger-type gripping mechanism
}

\author{
Jyh-Jone Lee *, Yu-Wei Tsai \\ Department of Mechanical Engineering, National Taiwan University, 1, Section 4, Roosevelt Road, Taipei \\ 106, Taiwan, ROC
}

Received 14 July 2004; received in revised form 19 January 2005; accepted 11 February 2005

Available online 13 April 2005

\begin{abstract}
In this paper, the path synthesis of a gripper-object mechanism with two rolling pairs is studied. The design equations of the mechanism used as a path generator are established. The dimension of set of admissible solutions in the kinematic synthesis is also discussed. It is shown that the design equations are transcendental and the optimization-based numerical technique is applied to solve the design equations. The synthesized solutions are illustrated to discuss the kinematic states of the mechanism. It is also shown that the optimization-based method is effective in finding the admissible synthesis solution of the mechanism. (C) 2005 Elsevier Ltd. All rights reserved.
\end{abstract}

Keywords: Gripper-object mechanism; Rolling pairs; Path synthesis; Optimization

\section{Introduction}

The finger-type gripping mechanisms as shown in Fig. 1 have been widely used for the industrial robots. In the figure, the finger-type gripping mechanism is composed of two links, each pinjointed at the ground link. The kinematic and force analyses of such mechanisms have been well investigated by numerous researchers [1-3]. Vassura and Nerozzi [4] showed a primitive study of object gripping operations with various positions and orientations using a multi-finger-type gripping mechanism. When gripping action begins, the object is gripped by the two links and if we

\footnotetext{
${ }^{*}$ Corresponding author.

E-mail address: jjlee@ccms.ntu.edu.tw (J.-J. Lee).
} 


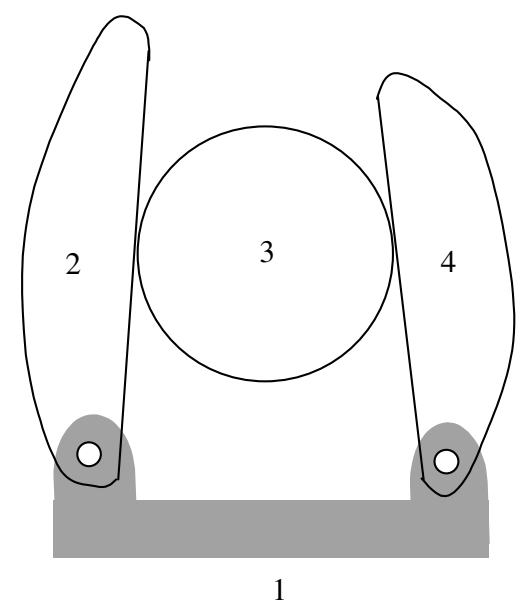

Fig. 1. A gripper-object mechanism.

assume that the contact between the link and the object is pure rolling, the gripper-object mechanism forms a four-link mechanism with one degree of freedom. Thus, a limited manipulation of the object can be obtained through the motion of the two cranks. Moreover, in certain application, the object may be required to pass through several prescribed positions and/or orientations to complete a given task from the engineering point of view. The kinematic analysis of the fourlink mechanism with pure rolling pairs has been studied by [5-7] or in a related field of multijointed dexterous hands design [8-10]. However, to our knowledge the kinematic synthesis of such mechanism was not studied before. In this work, the kinematic synthesis of a gripper-object mechanism is presented. The goal is to determine the design parameters of the mechanism to meet a given set of positions of the object. First, the design equations are derived using the complex number method. Then, the optimization based technique for solving the design equations are investigated. It is shown that the maximum number of precision points is eight. Three numerical examples are given to demonstrate the method of solution.

\section{Design equations}

As shown in Fig. 2, the gripper-object mechanism constitutes a four-link mechanism of which the two fingers are pin-jointed at the ground and each link contacts with the object in pure rolling. For the sake of simplicity, it is assumed that the object has a circular shape of radius $r$. As we are interested in the mechanism used for path generation, a vector $\mathbf{Z}_{2}$ can be defined as the vector from the pivot of link 2, $O_{a}$, to the contact point A between link 2 and link 3 (object) and a vector $\mathbf{Z}_{3}$ as the vector from point $A$ to the center of link 3 . On the other side of the mechanism, a vector $\mathbf{Z}_{5}$ is defined as the vector from the pivot of link $4, O_{b}$, to the contact point B between link 4 and link 3 and a vector $\mathbf{Z}_{6}$ as the vector from point $\mathbf{B}$ to the center of link 3 . Since a point on the object will be moved through a sequence of prescribed position by the two links, a coupler point on link 3 can be defined as a vector $\mathbf{Z}_{7}$ from the center of link 3. Then, vector-loop equations for the coupler point on link 3 with respect to the reference coordinate system can be written at every position as 


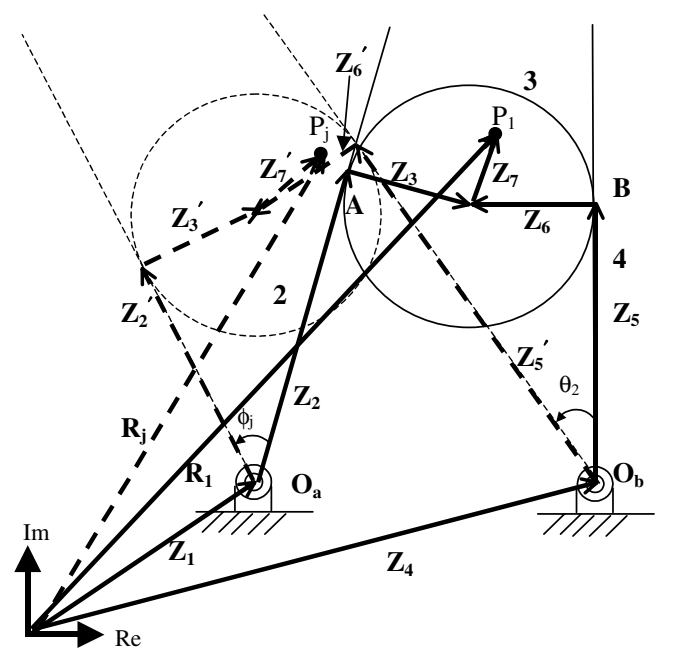

Fig. 2. Vector definition for the four-link mechanism.

$$
\begin{aligned}
& \mathbf{Z}_{1}+\mathbf{Z}_{2}+\mathbf{Z}_{3}+\mathbf{Z}_{7}=\mathbf{R}_{1} \\
& \mathbf{Z}_{4}+\mathbf{Z}_{5}+\mathbf{Z}_{6}+\mathbf{Z}_{7}=\mathbf{R}_{1}
\end{aligned}
$$

Here, $\mathbf{Z}_{1}-\mathbf{Z}_{7}$ are vectors representing the mechanism in its first reference position and $\mathbf{R}_{1}$ is the first position vector of the coupler point on link 3 . Then, after some movement the $j$ th position vector-loop equations can be written as

$$
\begin{aligned}
& \mathbf{Z}_{1}+\mathbf{Z}_{2}^{\prime}+\mathbf{Z}_{3}^{\prime}+\mathbf{Z}_{7}^{\prime}=\mathbf{R}_{j} \\
& \mathbf{Z}_{4}+\mathbf{Z}_{5}^{\prime}+\mathbf{Z}_{6}^{\prime}+\mathbf{Z}_{7}^{\prime}=\mathbf{R}_{j}
\end{aligned}
$$

where vector $\mathbf{Z}_{k}^{\prime}$ is the $j$ th position of the vector $\mathbf{Z}_{k}$ and $\mathbf{R}_{j}$ the $j$ th position vector of the coupler point. By subtracting Eq. (1) from Eq. (2), the path-increment equations can be written as

$$
\begin{aligned}
& \left(\mathbf{Z}_{2}^{\prime}-\mathbf{Z}_{2}\right)+\left(\mathbf{Z}_{3}^{\prime}-\mathbf{Z}_{3}\right)+\left(\mathbf{Z}_{7}^{\prime}-\mathbf{Z}_{7}\right)=\boldsymbol{\delta}_{j} \\
& \left(\mathbf{Z}_{5}^{\prime}-\mathbf{Z}_{5}\right)+\left(\mathbf{Z}_{6}^{\prime}-\mathbf{Z}_{6}\right)+\left(\mathbf{Z}_{7}^{\prime}-\mathbf{Z}_{7}\right)=\boldsymbol{\delta}_{j}
\end{aligned}
$$

where the vector $\boldsymbol{\delta}_{j}$ is defined as $\boldsymbol{\delta}_{j}=\mathbf{R}_{j}-\mathbf{R}_{1}$, and where $\phi_{j}, \alpha_{j}, \theta_{j}$ are, respectively, the angles of link 2, 3, and 4 measured with respect to their own reference position. Accordingly, when the gripper-object mechanism is manipulated vectors $\mathbf{Z}_{2}^{\prime}$ and $\mathbf{Z}_{5}^{\prime}$ will change both direction and magnitude, while $\mathbf{Z}_{3}^{\prime}, \mathbf{Z}_{6}^{\prime}$, and $\mathbf{Z}_{7}^{\prime}$ change only direction. Therefore, it is necessary to establish their kinematic relations during the motion. A free body diagram showing the kinematic relation between $\mathbf{Z}_{2}$ and $\mathbf{Z}_{2}^{\prime}$ is depicted in Fig. 3. Let $\beta_{j}$ be the angular displacement that the object rolls with respect to link 2 when link 2 rotates an angle $\phi_{j}$. Then the magnitude of vector $\mathbf{Z}_{2}^{\prime}$ can be obtained from the original magnitude of $\mathbf{Z}_{2}$ subtracted by the distance that link 3 has rolled on the link 2 . This yields

$$
\mathbf{Z}_{2}^{\prime}=\mathbf{Z}_{2} \mathrm{e}^{\mathrm{i} \phi_{j}}\left(1-\beta_{j}\left|\mathbf{Z}_{3}\right| /\left|\mathbf{Z}_{2}\right|\right)
$$




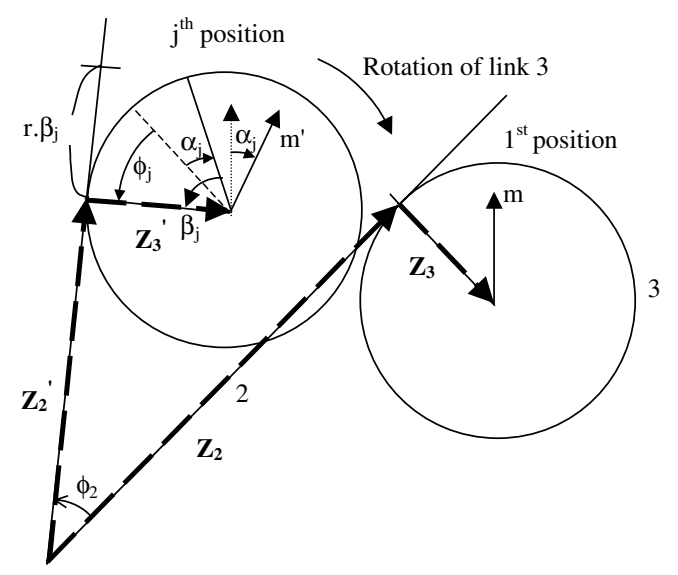

Fig. 3. Relations among angular displacements.

And since vector $\mathbf{Z}_{3}$ is so defined that it is always perpendicular to $\mathbf{Z}_{2}$, the following relations can be obtained

$$
\begin{aligned}
& \mathbf{Z}_{3}=\mathbf{Z}_{2} \mathrm{e}^{\mathrm{i}(-\pi / 2)}\left(\left|\mathbf{Z}_{3}\right| /\left|\mathbf{Z}_{2}\right|\right) \\
& \mathbf{Z}_{3}^{\prime}=\mathbf{Z}_{3} \mathrm{e}^{\mathrm{i} \phi_{j}}=-i \mathbf{Z}_{2}\left(\left|\mathbf{Z}_{3}\right| /\left|\mathbf{Z}_{2}\right|\right) \mathrm{e}^{\mathrm{i} \phi_{j}}
\end{aligned}
$$

Similarly, $\mathbf{Z}_{7}^{\prime}$ can be written as

$$
\mathbf{Z}_{7}^{\prime}=\mathbf{Z}_{7} \mathrm{e}^{\mathrm{i} \alpha_{j}}
$$

Note that we have introduced the variable $\beta_{j}$ in Eq. (4). This variable can be further related to the combined motion of link 2 and 3 from the superposition viewpoint. As shown in Fig. 3, the initial position of link 3 is given by a line marked with an arrow $m$ directing from the center to a designated direction. After some rotation, link 3 is moved to the new position while the mark is moved to $m^{\prime}$. The angular motion of link 3 as given by the angle between $\mathrm{m}$ and $m^{\prime}$ can be obtained as follows. First fix link 3 to link 2 as a whole link and rotate an angle $\phi_{j}$ and then move link 3 an angle $\beta$ with respect to link 2 . The sum of the above angular displacements will give the resultant angular displacement of link 3 . This yields

$$
\beta_{j}=\alpha_{j}-\phi_{j}
$$

Substituting Eq. (7) into Eq. (4) to suppress $\beta_{j}$ and again back substituting Eqs. (4), (5a) and (5b) into (3a), yields

$$
\mathbf{Z}_{2}\left(\mathrm{e}^{\mathrm{i} \phi_{j}}-1\right)+\mathbf{Z}_{2} A\left[\boldsymbol{i}-i \mathrm{e}^{\mathrm{i} \phi_{j}}-\left(\phi_{j}-\alpha_{j}\right) \mathrm{e}^{\mathrm{i} \phi_{j}}\right]+\mathbf{Z}_{7}\left(\mathrm{e}^{\mathrm{i} \alpha_{j}}-1\right)=\boldsymbol{\delta}_{j}
$$

where $A=\left|\mathbf{Z}_{3}\right| /\left|\mathbf{Z}_{2}\right|$. Eq. (8a) is the path-increment vector equation for the left-hand part of the mechanism. Similarly, the path-increment vector equation for the right-hand part of the mechanism can be derived as

$$
\mathbf{Z}_{5}\left(\mathrm{e}^{\mathrm{i} \theta_{j}}-1\right)+\mathbf{Z}_{5} B\left[i \mathrm{e}^{\mathrm{i} \theta_{j}}-\boldsymbol{i}+\left(\theta_{j}-\alpha_{j}\right) \mathrm{e}^{\mathrm{i} \theta_{j}}\right]+\mathbf{Z}_{7}\left(\mathrm{e}^{\mathrm{i} \alpha_{j}}-1\right)=\boldsymbol{\delta}_{j}
$$


Table 1

The number of precision points vs. the dimension of solution sets

\begin{tabular}{llll}
\hline No. of positions & No. of scalar equations & No. of design variables & Dimension of the set of solutions \\
\hline 2 & 4 & $10\left(\mathbf{Z}_{2}, \mathbf{Z}_{5}, \mathbf{Z}_{7}, r, \phi_{2}, \theta_{2}, \alpha_{2}\right)$ & 6 (6 free choice) \\
3 & 8 & $13\left(\right.$ above $\left.+\phi_{3}, \theta_{3}, \alpha_{3}\right)$ & 5 \\
4 & 12 & $16\left(\right.$ above $\left.+\phi_{4}, \theta_{4}, \alpha_{4}\right)$ & 4 \\
5 & 16 & 19 & 3 \\
6 & 20 & 22 & 2 \\
7 & 24 & 25 & 1 \\
8 & 28 & 28 & 0 \\
\hline
\end{tabular}

where $B=\left|\mathbf{Z}_{6}\right| /\left|\mathbf{Z}_{5}\right|$. Letting $\mathbf{Z}_{2}=Z_{2 x}+i Z_{2 y}, \mathbf{Z}_{5}=Z_{5 x}+i Z_{5 y}, \mathbf{Z}_{7}=Z_{7 x}+i Z_{7 y}$ and $\boldsymbol{\delta}_{j}=\delta_{j x}+i \delta_{j y}$ and deploying the two complex number equations into scalar form, one yields

$$
\begin{aligned}
& {\left[Z_{2 x}+A Z_{2 y}-A Z_{2 x}\left(\phi_{j}-\alpha_{j}\right)\right] \cos \phi_{j}+\left[-Z_{2 y}+A Z_{2 x}+A Z_{2 y}\left(\phi_{j}-\alpha_{j}\right)\right] \sin \phi_{j}} \\
& \quad-Z_{2 x}-A Z_{2 y}+Z_{7 x} \cos \alpha_{j}-Z_{7 y} \sin \alpha_{j}-Z_{7 x}=\delta_{j x} \\
& \quad\left[Z_{2 y}-A Z_{2 x}-A Z_{2 y}\left(\phi_{j}-\alpha_{j}\right)\right] \cos \phi_{j}+\left[Z_{2 x}+A Z_{2 y}-A Z_{2 x}\left(\phi_{j}-\alpha_{j}\right)\right] \sin \phi_{j} \\
& \quad-Z_{2 y}+A Z_{2 x}+Z_{7 x} \sin \alpha_{j}+Z_{7 y} \cos \alpha_{j}-Z_{7 y}=\delta_{j y}
\end{aligned}
$$

Step 1

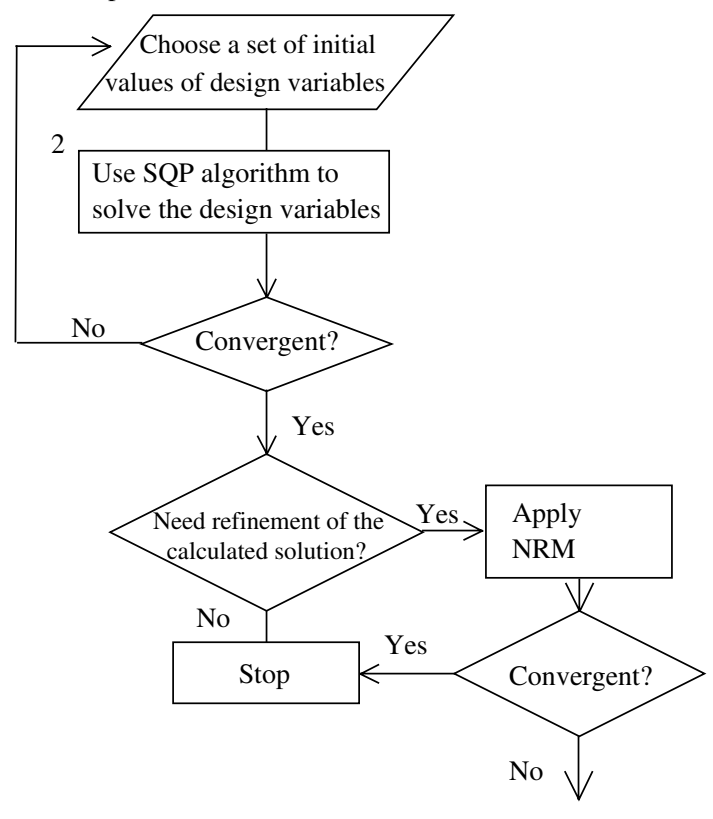

Go to Step 1

Fig. 4. Flow chart of the computational procedure. 


$$
\begin{aligned}
& {\left[Z_{5 x}-B Z_{5 y}+B Z_{5 x}\left(\theta_{j}-\alpha_{j}\right)\right] \cos \theta_{j}+\left[-Z_{5 y}-B Z_{5 x}-B Z_{5 y}\left(\theta_{j}-\alpha_{j}\right)\right] \sin \theta_{j}} \\
& \quad-Z_{5 x}+B Z_{5 y}+Z_{7 x} \cos \alpha_{j}-Z_{7 y} \sin \alpha_{j}-Z_{7 x}=\delta_{j x} \\
& {\left[Z_{5 y}+B Z_{5 x}+B Z_{5 y}\left(\theta_{j}-\alpha_{j}\right)\right] \cos \theta_{j}+\left[Z_{5 x}-B Z_{5 y}+B Z_{5 x}\left(\theta_{j}-\alpha_{j}\right)\right] \sin \theta_{j}} \\
& \quad-Z_{5 y}-B Z_{5 x}+Z_{7 x} \sin \alpha_{j}+Z_{7 y} \cos \alpha_{j}-Z_{7 y}=\delta_{j y}
\end{aligned}
$$

We summarize that in Eqs. (9a)-(9d), the design variables are $\mathbf{Z}_{2}, \mathbf{Z}_{5}, \mathbf{Z}_{7}, r\left(\left|\mathbf{Z}_{3}\right|\right), \phi_{j}, \theta_{j}$, and $\alpha_{j}$. By counting the number of design equations versus the number of design variables, we can obtain the dimension of the set of solutions for the path generation of the four-link mechanism with rolling pairs as listed in Table 1. Therefore, it can be concluded that the maximum number of precision points is eight. In view of Eqs. (9a)-(9d), because the equations contain the terms $\phi \cos \phi$ and $\phi$

\begin{tabular}{|l|l|}
\hline point & coordinate \\
\hline $\mathrm{P}_{1}$ & $(3.2577,2.8759)$ \\
\hline $\mathrm{P}_{2}$ & $(2.3955,3.1205)$ \\
\hline $\mathrm{P}_{3}$ & $(1.1909,3.1188)$ \\
\hline $\mathrm{P}_{4}$ & $(0.5430,2.9753)$ \\
\hline $\mathrm{P}_{5}$ & $(-0.6377,2.4843)$ \\
\hline
\end{tabular}

(a)

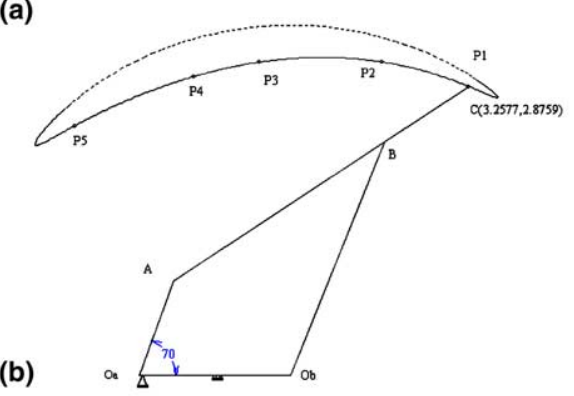

(b)

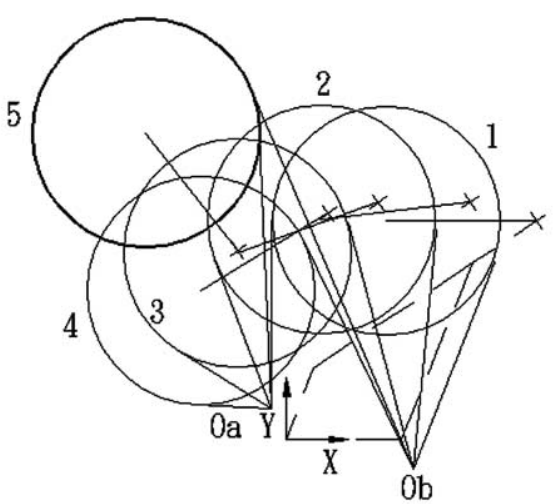

(c)

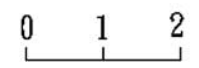

Fig. 5. (a) Numerical data for the five precision points. (b) Five precision points on a smooth curve generated by a hinged four-bar linkage $\left(O_{a} O_{b}=1.5, O_{a} A=1, A B=2.5, B C=1, O_{b} B=2.5\right)$. (c) Synthesized mechanism for Example 1. 
$\sin \phi$, the system of equations is transcendental and no closed form solutions can be found. Therefore, a numerical method needs to be developed to solve this system of nonlinear equations.

\section{Kinematic synthesis}

Many different numerical methods for solving the nonlinear kinematic equations were investigated in the past. A common method is to use the Newton-Raphson method (NRM) to solve nonlinear equations. However, the major drawback of this method is that it is sensitive to the starting estimate of the solution, i.e., if the starting estimate is not sufficiently accurate, this method usually does not converge. Further, there is always little information about the starting mechanism that can be provided for the kinematic synthesis problem. Another method is to use optimization based technique. The advantage of using the optimization method lies in that the starting vectors need not be very close to the final solution. In addition, the design constraints are easier to be dealt with provided that the problem is formulated in a constrained optimization form [11-13]. In this work, we shall in the first phase use the optimization-based algorithm to obtain an optimized mechanism with sufficient accuracy. Then, in the second phase, by using these optimized solutions as the starting mechanism, the solutions are refined by the Newton-Raphson method according to the desired degree of accuracy. The methodology is described as follows.

In order to solve Eqs. (9a)-(9d), an initial estimate of the unknown parameters is made. The approximate scalar equations of the path-increment of the mechanism are calculated as

Table 2

Initial estimate, optimized solution, and solution refined by NRM for Example 1

\begin{tabular}{llcc}
\hline & Initial & Optimized & NRM refined \\
\hline$Z_{2 x}$ & 1 & -0.0532868 & 0.000000 \\
$Z_{2 y}$ & 3 & 2.4377260 & 2.4610662 \\
$Z_{5 x}$ & 1 & 1.3490103 & 1.0458503 \\
$Z_{5 y}$ & 3 & 3.4455074 & 2.7159663 \\
$Z_{7 x}$ & 1 & 1.5295531 & 1.9517701 \\
$Z_{7 y}$ & 1 & -0.5388531 & 0.000000 \\
$\phi_{2}$ & 0.5 & 0.4027096 & 0.3680146 \\
$\phi_{3}$ & 1.5 & 1.1179636 & 1.0181245 \\
$\phi_{4}$ & 2.5 & 1.6458459 & 1.5274409 \\
$\phi_{5}$ & 3.5 & 0.23868652 & 3.1843420 \\
$\theta_{2}$ & 0.2 & 0.5617761 & 0.2752819 \\
$\theta_{3}$ & 0.4 & 0.7392133 & 0.6270665 \\
$\theta_{4}$ & 0.6 & 0.8970190 & 0.8141967 \\
$\theta_{5}$ & 0.7 & 0.14426801 & 0.7715247 \\
$\alpha_{2}$ & 0.3 & 0.4658503 & 0.1149596 \\
$\alpha_{3}$ & 0.6 & 0.7453434 & 0.3469881 \\
$\alpha_{4}$ & 0.9 & -0.0887144 & 0.5491520 \\
$\alpha_{5}$ & 1.2 & $2.96671795 \mathrm{e}-005$ & -0.9009955 \\
Obj. $f$ & & & $1.0 \mathrm{e}-025$ \\
\hline
\end{tabular}




$$
\begin{aligned}
\mathrm{d} L_{j x}= & {\left[\left(Z_{2 x}\right)^{*}+A^{*}\left(Z_{2 y}\right)^{*}-A^{*}\left(Z_{2 x}\right)^{*}\left(\phi_{j}^{*}-\alpha_{j}^{*}\right)\right] \cos \phi_{j}^{*}+\left[-\left(Z_{2 y}\right)^{*}+A^{*}\left(Z_{2 x}\right)^{*}\right.} \\
& \left.+A^{*}\left(Z_{2 y}\right)^{*}\left(\phi_{j}^{*}-\alpha_{j}^{*}\right)\right] \sin \phi_{j}^{*}-\left(Z_{2 x}\right)^{*}-A^{*}\left(Z_{2 y}\right)^{*}+\left(Z_{7 x}\right)^{*} \cos \alpha_{j}^{*}-\left(Z_{7 y}\right)^{*} \sin \alpha_{j}^{*}-\left(Z_{7 x}\right)^{*} \\
\mathrm{~d} L_{j y}= & {\left[\left(Z_{2 y}\right)^{*}-A^{*}\left(Z_{2 x}\right)^{*}-A^{*}\left(Z_{2 y}\right)^{*}\left(\phi_{j}^{*}-\alpha_{j}^{*}\right)\right] \cos \phi_{j}^{*}+\left[\left(Z_{2 x}\right)^{*}+A^{*}\left(Z_{2 y}\right)^{*}\right.} \\
& \left.-A^{*}\left(Z_{2 x}\right)^{*}\left(\phi_{j}^{*}-\alpha_{j}^{*}\right)\right] \sin \phi_{j}^{*}-\left(Z_{2 y}\right)^{*}+A^{*}\left(Z_{2 x}\right)^{*}+\left(Z_{7 x}\right)^{*} \sin \alpha_{j}^{*}+\left(Z_{7 y}\right)^{*} \cos \alpha_{j}^{*}-\left(Z_{7 y}\right)^{*} \\
\mathrm{~d} R_{j x}= & {\left[\left(Z_{5 x}\right)^{*}-B^{*}\left(Z_{5 y}\right)^{*}+B^{*}\left(Z_{5 x}\right)^{*}\left(\theta_{j}^{*}-\alpha_{j}^{*}\right)\right] \cos \theta_{j}^{*}+\left[-\left(Z_{5 y}\right)^{*} B^{*}\left(Z_{5 x}\right)^{*}\right.} \\
& \left.-B^{*}\left(Z_{5 y}\right)^{*}\left(\theta_{j}^{*}-\alpha_{j}^{*}\right)\right] \sin \theta_{j}^{*}-\left(Z_{5 x}\right)^{*}+B^{*}\left(Z_{5 y}\right)^{*}+\left(Z_{7 x}\right)^{*} \cos \alpha_{j}^{*}-\left(Z_{7 y}\right)^{*} \sin \alpha_{j}^{*}-\left(Z_{7 x}\right)^{*} \\
\mathrm{~d} R_{j y}= & {\left[\left(Z_{5 y}\right)^{*}+B^{*}\left(Z_{5 x}\right)^{*}+B^{*}\left(Z_{5 y}\right)^{*}\left(\theta_{j}^{*}-\alpha_{j}^{*}\right)\right] \cos \theta_{j}^{*}+\left[\left(Z_{5 x}\right)^{*}-B^{*}\left(Z_{5 y}\right)^{*}\right.} \\
& \left.+B^{*}\left(Z_{5 x}\right)^{*}\left(\theta_{j}^{*}-\alpha_{j}^{*}\right)\right] \sin \theta_{j}^{*}-\left(Z_{5 y}\right)^{*}-B^{*}\left(Z_{5 x}\right)^{*}+\left(Z_{7 x}\right)^{*} \sin \alpha_{j}^{*} \\
& +\left(Z_{7 y}\right)^{*} \cos \alpha_{j}^{*}-\left(Z_{7 y}\right)^{*} \quad j=2,3, \ldots, n
\end{aligned}
$$

where $\left(\mathrm{d} L_{j x}, \mathrm{~d} L_{j y}\right)$ represents the $x$ and $y$ components of the left-hand path-increment, $\left(\mathrm{d} R_{j x}, \mathrm{~d} R_{j y}\right)$ the $x$ and $y$ components of the right-hand path-increment of the mechanism, and (\#) ${ }^{*}$ the approx-

Table 3

A second solution for Example 1

\begin{tabular}{lccl}
\hline & Initial & Optimal & NRM refined \\
\hline$Z_{2 x}$ & 1 & 3.0406893 & 0.7427273 \\
$Z_{2 y}$ & 5 & 3.2576885 & 3.2339385 \\
$Z_{5 x}$ & -1 & 0.9490885 & 1.1388037 \\
$Z_{5 y}$ & 5.7495008 & 2.8572640 \\
$Z_{7 x}$ & 5 & 0.5676883 & 2.2864945 \\
$Z_{7 y}$ & 1 & 0.8345637 & 0.1568637 \\
$\phi_{2}$ & 1 & 0.2533224 & 0.2750532 \\
$\phi_{3}$ & 0.5 & 0.6444206 & 0.7164374 \\
$\phi_{4}$ & 1.5 & 0.8979535 & 1.0146111 \\
$\phi_{5}$ & 2.5 & 1.5493414 & 1.8287129 \\
$\theta_{2}$ & 3.5 & 0.1706180 & 0.2667330 \\
$\theta_{3}$ & 0.3660592 & 0.5974675 \\
$\theta_{4}$ & 0.5 & 0.4623882 & 0.7723353 \\
$\theta_{5}$ & 0.6 & 0.6376358 & 1.1158335 \\
$\alpha_{2}$ & 0.7 & -0.1962117 & 0.0441404 \\
$\alpha_{3}$ & 0.2 & -0.3801050 & 0.1267436 \\
$\alpha_{4}$ & 0.3 & -0.4335097 & 0.2015597 \\
$\alpha_{5}$ & 0.4 & -0.4077390 & 0.4861544 \\
Obj. $f$ & 0.5 & $2.83505636 \mathrm{e}-005$ & $1.51 \mathrm{e}-006$ \\
\hline
\end{tabular}


imate value of (\#). To obtain the optimal values of the design variables, one can define the objective function as the sum of the squares of the difference between each component of the approximate path-increment vector $\left(\mathrm{d} L_{j x}, \mathrm{~d} L_{j y}\right)$ and the desired path increment vector $\left(\delta_{j x}, \delta_{j y}\right)$, which can be written as

$$
f=\sum_{j}^{n}\left[\left(\mathrm{~d} L_{j x}-\delta_{j x}\right)^{2}+\left(\mathrm{d} L_{j y}-\delta_{j y}\right)^{2}\right]
$$

In the meantime, both the approximate left-hand and right-hand path-increments must be compatible, i.e., they are subject to the equality constraints

$$
\begin{aligned}
& \mathrm{d} L_{j x}-\mathrm{d} R_{j x}=0 \\
& \mathrm{~d} L_{j y}-\mathrm{d} R_{j y}=0 \quad j=2,3, \ldots, n
\end{aligned}
$$

The about constraints also imply the loop closure formed by the vectors in the new position and original position remains closed. The procedure can now be summarized as

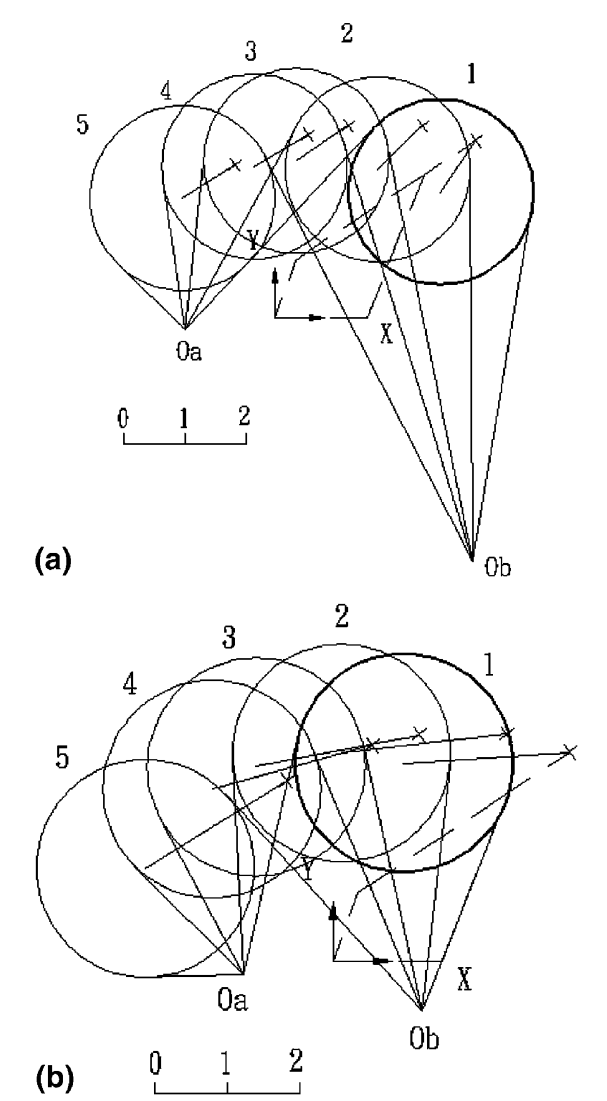

Fig. 6. (a) A second solution by optimization for Example 1. (b) A second solution by NRM for Example 1. 


\section{First phase}

(i) State the problem as

Minimize $f=\sum_{j}^{n}\left[\left(\mathrm{~d} L_{j x}-\delta_{j x}\right)^{2}+\left(\mathrm{d} L_{j y}-\delta_{j y}\right)^{2}\right]$

Subject to

$$
\begin{aligned}
& \mathrm{d} L_{j x}-\mathrm{d} R_{j x}=0 \\
& \mathrm{~d} L_{j y}-\mathrm{d} R_{j y}=0
\end{aligned}
$$

where $\mathrm{d} L_{j x}, \mathrm{~d} L_{j y}, \mathrm{~d} R_{j x}$, and $\mathrm{d} R_{j y}$ are given in Eq. (10).

(ii) If necessary, add estimated ranges of the unknown variables to the optimization problem as inequality constraint. These can be expressed as

$c_{1} \leqslant Z_{2 x} \leqslant d_{1}$

$c_{2} \leqslant Z_{2 y} \leqslant d_{2}$

$c_{3} \leqslant Z_{3 x} \leqslant d_{3}$

:

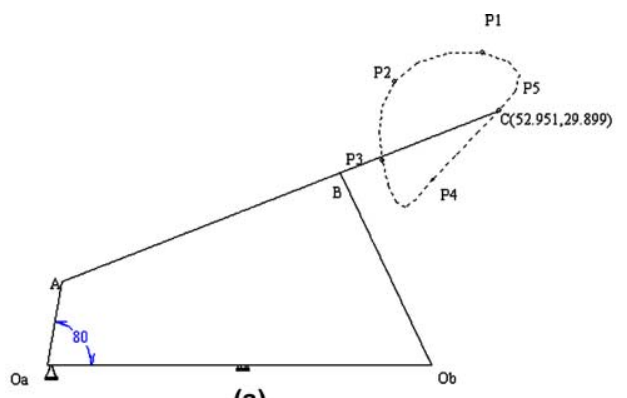

(a)

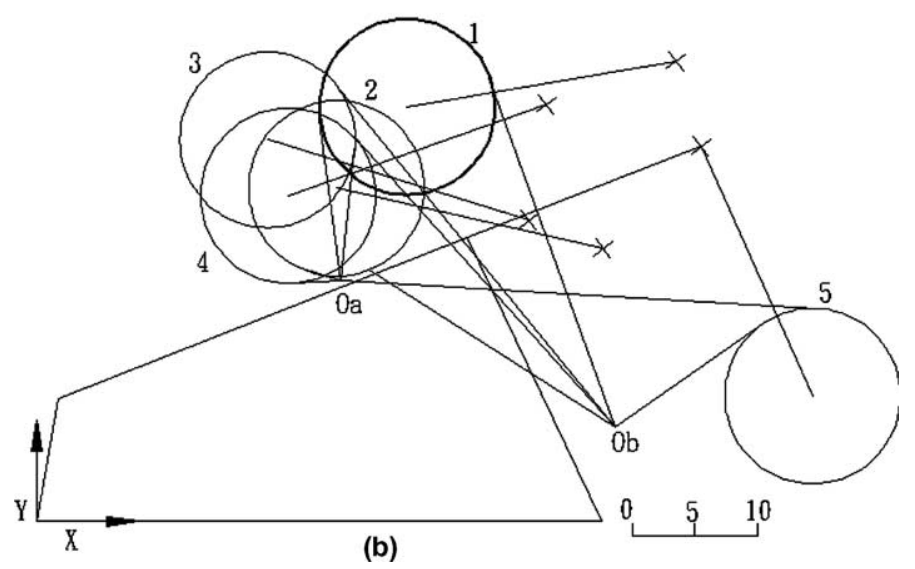

Fig. 7. (a) Five precision points on a looped curve $\left(O_{a} O_{b}=45, O_{a} A=10, A B=35, B C=20, O_{b} B=25\right)$, Example 2. (b) Synthesized mechanism for Example 2. 
where $c_{i}^{\prime}$ s and $d_{i}^{\prime}$ 's are the lower- and upper-bound values. Adding the inequality constraints may allow the optimization algorithm to search in a smaller region and result in more efficiency than an unconstrained problem. Besides, with specified regions, it is also of help in identifying multiple solutions.

(iii) Solve the constrained optimization problem by using the optimization algorithm. In this work, the sequential quadratic programming (SQP) algorithm is utilized to solve the problem since it allows us to closely mimic Newton's method for constrained optimization. Detailed algorithms of SQP can be found in [14-16].

\section{Second phase}

(iv) Refine the solution by using the NRM, if necessary. The accuracy and convergence of the optimization may depend upon the complexity of the equations. A trade-off needs to be made between the two factors. For our experience, to run with a tight accuracy algorithm, the problem solving often tends to become slow or not convergent. Therefore, in this study, the tolerance on the objective function value is necessarily set to be a reasonable value. Once it converges, the optimized solution can be further refined by using the NRM with higher accuracy. Otherwise, a new set of estimate is reassigned and the whole procedure is repeated. A flow chart of the computational procedure is also shown in Fig. 4.

Table 4

Initial estimate, optimized solution, and solution refined by NRM for Example 2

\begin{tabular}{llrr}
\hline & Initial & Optimized & NRM refined \\
\hline$Z_{2 x}$ & 15 & -1.7839231 & -1.6747586 \\
$Z_{2 y}$ & 15 & 12.9798512 & 12.9426222 \\
$Z_{5 x}$ & -10.1171609 & -10.0109052 \\
$Z_{5 y}$ & 10 & 27.8404824 & 27.8193140 \\
$Z_{7 x}$ & 15 & 21.7077213 & 21.4798428 \\
$Z_{7 y}$ & 5 & 3.7006696 & 3.6639345 \\
$\phi_{2}$ & 5 & 1.4712161 & 1.4950185 \\
$\phi_{3}$ & 0.5 & 9.1999943 & 9.1877197 \\
$\phi_{4}$ & 1.5 & 8.0754557 & 8.0958396 \\
$\phi_{5}$ & 2.5 & 4.5145023 & 4.5263997 \\
$\theta_{2}$ & 3.5 & 0.3880738 & 0.3883076 \\
$\theta_{3}$ & 0.3 & 6.6294514 & 6.6220890 \\
$\theta_{4}$ & 0.6 & -2.3770247 & -2.3822744 \\
$\theta_{5}$ & 0.9 & 1.8125847 & 1.8321897 \\
$\alpha_{2}$ & 1.3 & 0.1711817 & 0.1713525 \\
$\alpha_{3}$ & 0.3 & 5.8357809 & 5.8156819 \\
$\alpha_{4}$ & 0.6 & 5.9047050 & 5.8917843 \\
$\alpha_{5}$ & 0.9 & 8.0987116 & 8.1068304 \\
Obj. $f$ & 1.3 & $2.22709126 \mathrm{e}-007$ & $1.732 \mathrm{e}-007$ \\
\hline
\end{tabular}



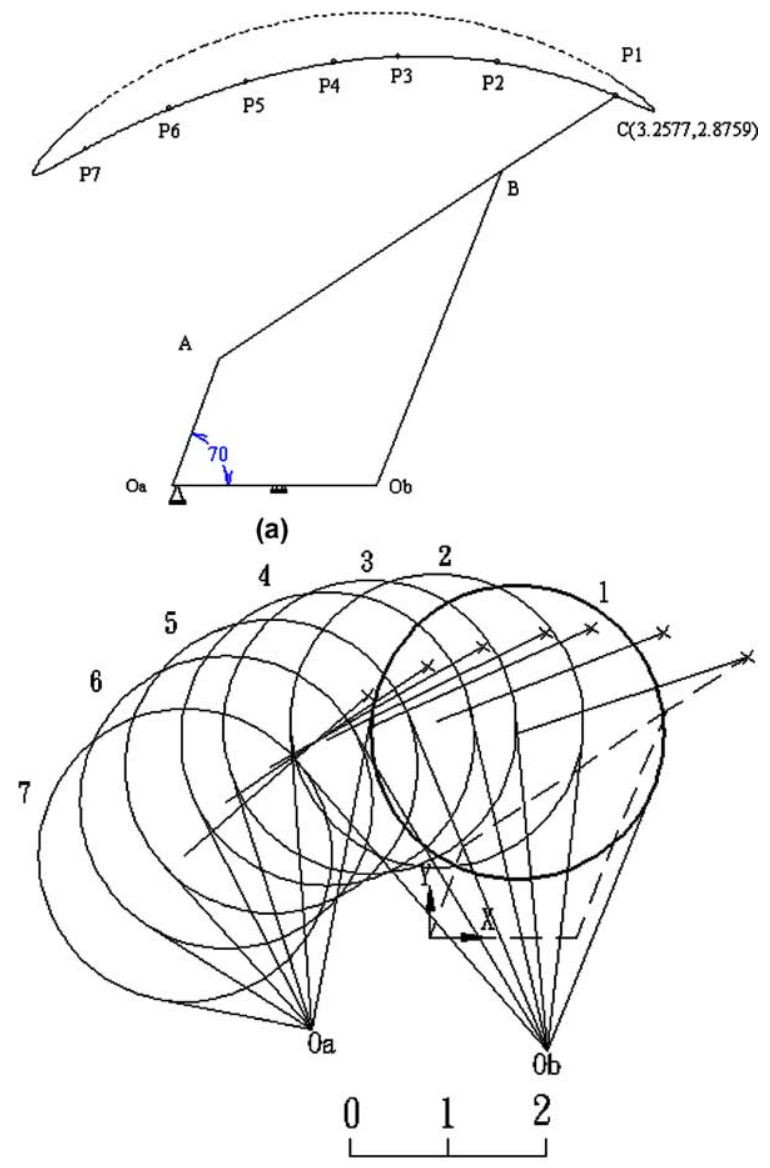

(b)

Fig. 8. (a) Seven precision points chosen from a smooth curve. (b) Synthesized mechanism for seven precision points.

\section{Numerical examples}

In order to illustrate this technique, three examples are given here. We will demonstrate the procedure by five-point and seven-point synthesis problems.

Example 1 (Synthesis of five-point on a smooth curve). For the purpose of illustration, we select the precision points from the coupler curve of a hinged four-bar mechanism rather than specifying them randomly. Fig. 5a lists the five desired precision points taken from a smooth segment of coupler curve of a hinged four-bar mechanism shown in Fig. 5b. Let the radius of circular object be $r=1.5$, and design variables as unconstrained. We start a set of initial estimates as listed in the first column of Table 2. The initial values are chosen somewhat in a trial-and-error manner. The tolerance on the objective function value and the number of iterations is set as $1.0 \mathrm{E}-6$ and 10,000 times, respectively. The optimization result is shown in the second column of Table 2. A refined solution run by NRM is also listed in Table 2 for reference. Fig. 5c shows the synthesized mechanism associated with the configuration at each precision position. Notice that the 
Table 5

Initial estimate, optimized solution, and solution refined by NRM for Example 3

\begin{tabular}{llll}
\hline & Initial & Optimized & NRM refined \\
\hline$Z_{2 x}$ & 0.742727 & 0.740479 & 0.6219171 \\
$Z_{2 y}$ & 3.233938 & 3.212795 & 3.3128858 \\
$Z_{5 x}$ & 1.138804 & 1.139126 & 1.0799731 \\
$Z_{5 y}$ & 2.857264 & 2.846242 & 2.6944957 \\
$Z_{7 x}$ & 2.286494 & 2.274580 & 2.3667623 \\
$Z_{7 y}$ & 0.156864 & 0.144418 & 0.7854626 \\
$\phi_{2}$ & 0.275053 & 0.277115 & 0.2571668 \\
$\phi_{3}$ & 0.500000 & 0.535710 & 0.4937091 \\
$\phi_{4}$ & 0.716437 & 0.723275 & 0.6618354 \\
$\phi_{5}$ & 1.014611 & 1.026003 & 0.9237036 \\
$\phi_{6}$ & 1.300000 & 1.359954 & 1.1949263 \\
$\phi_{7}$ & 1.828713 & 1.863492 & 1.5623963 \\
$\theta_{2}$ & 0.266733 & 0.267595 & 0.2697532 \\
$\theta_{3}$ & 0.400000 & 0.472223 & 0.4756289 \\
$\theta_{4}$ & 0.597468 & 0.599494 & 0.6025546 \\
$\theta_{5}$ & 0.772335 & 0.775066 & 0.7743973 \\
$\theta_{6}$ & 0.900000 & 0.934307 & 0.9253102 \\
$\theta_{7}$ & 1.115834 & 1.120417 & 1.0944705 \\
$\alpha_{2}$ & 0.044140 & 0.044523 & 0.0510131 \\
$\alpha_{3}$ & 0.080000 & 0.089591 & 0.0964186 \\
$\alpha_{4}$ & 0.126744 & 0.128488 & 0.1312368 \\
$\alpha_{5}$ & 0.201560 & 0.205214 & 0.1942089 \\
$\alpha_{6}$ & 0.300000 & 0.309926 & 0.2731399 \\
$\alpha_{7}$ & 0.486154 & 0.499195 & 0.3996746 \\
Obj. $f$ & & $8.883984 \mathrm{e}-006$ & $3.55021 \mathrm{e}-009$ \\
\hline
\end{tabular}

mechanism will need to change its assembly condition when transiting from position 4 to position 5 as is the branch problem occurred in the conventional synthesis of hinged four-bar mechanism. To search for other admissible solutions, we now reassign another set of initial values and constrain all angles between 0 and $2 \pi$. The procedure is first run by the optimization algorithm and then refined by NRM. The numerical results are listed in Table 3 while the synthesized mechanism sketched from the optimization and NRM refined values is, respectively, shown in Fig. 6a and b. Note that the two mechanisms by the optimization result and NRM are different. This implies that both methods converge to their individual local minimum.

Example 2 (Synthesis of five-point distributed along a looped curve). In this example, we choose the five points distributed along a looped curve in which three points are on one side and the rest two points are on another side of the loop as shown in Fig. 7a. The coordinates for the points are $P_{1}(50.9694,36.6948), P_{2}(40.6130,33.2529), P_{3}(39.2307,24.0298), P_{4}(45.1568,21.7685)$, and $P_{5}$ (52.9514,29.8988). This is in contrast to Example 1 where the five points are lying on the smooth part of the coupler curve. Again, it is assumed that $r=7$ and the joint angles are left 
unconstrained. The initial estimate, optimized solution, and refined solution by Newton-Raphson method are listed in Table 4. Fig. $7 \mathrm{~b}$ also shows the synthesized mechanism associated with the configuration at each precision position. Note that the values of $\phi_{3}$ and $\phi_{4}$ exceed $2 \pi$, indicating the branch problem occurs during the motions from position 2 to 3 and 3 to 4 .

Example 3 (Synthesis of seven-point on a smooth curve). In this example, we apply the technique for the seven-precision-point synthesis. It is far difficult to solve for the seven-precision-point problem since the number of nonlinear equations has been increased up to twenty four. To illustrate, we still use the same segment of the coupler curve in Example 1 for generating the precision points. Along the path curve, two more precision points, points $P_{3}(1.6627,3.1630)$ and $P_{6}$ $(-0.0223,2.7773)$ in Fig. 8a, together with the previous five precision points are chosen for the seven-precision-point synthesis. We then use the 18 values obtained in the five-point synthesis as the initial estimate for the seven-point problem. In such condition, only six more newly estimated values are needed to run the optimization procedure. This lessens the difficulty in making a blind guess about all the initial values. The final solution converges as shown in Table 5. The synthesized mechanism and its configuration at each precision point are shown in Fig. 8b. In this example, the mechanism found is not far from the initial values.

\section{Discussion}

There exist many other numerical methods for solving a system of nonlinear equations. As with most numerical iteration methods, for this problem the optimization methodology does not guarantee finding admissible solutions given a set of initial estimate. In some cases, we applied the NRM followed by the optimization result and found that the NRM may present a better solution from practicality point of view. This implies that there may exist many local solutions for the system of equations. Nonetheless, the optimization methodology does diminish the burden of guessing the 'right' initial values. In this work, we have also tried searching algorithms by general genetic algorithm [17] and cyclic coordinate descent (CCD) method [18,19]. Our experience is that the CCD method does not converge the initial values so well as the optimization based method, since it changes only one variable at a time to minimize the objective function while the general genetic searching algorithm is not so efficient and most of the time yields no solution for our problem. In spite of these, studies of using more advanced genetic searching algorithm may need further exploration. Another advantage of using the optimization based method is that adding upper/lower bounds for design variables as inequality constraints are easily treated in the formulation. Especially, they are helpful in searching for multiple solutions when the regions of design parameters are specified, as shown in Example 1.

\section{Conclusion}

A methodology for solving the kinematic synthesis of gripper-object mechanism with rolling pairs has been developed. Design equations for the kinematic synthesis are derived. It is shown 
that up to eight precision positions of the object can be prescribed. Further, the solution method for the synthesis is also described. Numerical examples are given to demonstrate the design methodology.

\section{Acknowledgement}

The financial support of the National Science Council (NSC-93-2212-E-002-056) of Taiwan is gratefully appreciated.

\section{References}

[1] F.Y. Chen, Gripping mechanism for industrial robot grippers, Mechanism and Machine Theory 17 (5) (1982) 299311.

[2] F.Y. Chen, Force analysis and design considerations of grippers, The industrial robot (1982) 243-249, December.

[3] D.T. Pham, W.B. Hagmbotham, Robot Gripper, IFS (Publications) Ltd., UK, 1986.

[4] G. Vassura, A. Nerozzi, A multi-finger gripper, in: Proceedings of the 10th International Symposium on Industrial Robots, Milan, Italy, 1980 (also reprinted in Ref. [3]).

[5] A. Cole, J. Hauser, S. Sastry, Kinematics and control of multifingered hands with rolling contact, in: Proceedings of IEEE International Conference on Robotics and Automation, 1988, pp. 228-233.

[6] S.K. Agrawal, R. Pandravada, Kinematics and workspace of a rolling disk between planar manipulators, in: Proceedings of the American Control Conference, San Francisco, CA, June, 1993, pp. 741-745.

[7] W. Chen, V. Kumar, Workspace of planar cooperating robots with rolling contacts, Advanced Robotics, The International Journal of the Robotics Society of Japan 9 (5) (1995) 483-504.

[8] T. Okada, Computer control of multi-jointed finger system, in: Proceedings of the Sixth International Joint Conference on Artificial Intelligence, Tokyo, Japan, 1979.

[9] J.K. Salisbury, Kinematic and force analysis of articulated hands, Ph.D. Thesis, Department of Mechanical Engineering, Stanford University, Stanford, California, USA, 1982.

[10] S.C. Jacobsen, J.E. Wood, D.F. Knutti, K.B. Biggers, The UTAH/MIT dexterous hand, International Journal of Robotics Research 3 (4) (1984) 21-50.

[11] R.L. Fox, K.D. Willmert, Optimum design of curve-generating linkage with inequality constraints, Transaction of the ASME B 89 (1) (1967) 144-152.

[12] S. Mahalingam, A.M. Sharan, The nonlinear displacement analysis of robotic manipulators using the complex optimization method, Mech. Mach. Theory 22 (1) (1987) 89-95.

[13] P. Premkumar, S.N. Kramer, Position, velocity and acceleration synthesis of the RRSS spatial path generating mechanism using the selective precision synthesis method, ASME Transactions, Journal of Mechanisms, Transmissions and Automation in Design 111 (1) (1989) 54-58.

[14] J.S. Arora, Introduction to Optimum Design, McGraw-Hill, New York, 1989.

[15] P. Venkataraman, Applied Optimization with MATLAB Programming, John Wiley and Sons, New York, 2002.

[16] Optimization Toolbox User's Guide, Version 2, The MathWorks Inc. (2000).

[17] D.E. Goldberg, Genetic Algorithms in Search Optimization and Machine Learning, Addison-Wesley, USA, 1989.

[18] L.T. Wang, C.C. Chen, A combined optimization method for solving the inverse kinematics problem of mechanical manipulators, IEEE Transactions on Robotics and Automation 7 (4) (1991) 489-499.

[19] L.T. Wang, C.C. Chen, On the numerical kinematic analysis of general parallel robotic manipulators, IEEE Transactions on Robotics and Automation 9 (3) (1993) 489-499. 